This is the post print version of the article Kertész, Turunen: What Can We Learn from the Long-Term Users of a Social

9th International Conference, ICSR 2017, Tsukuba, Japan,

November 22-24, 2017, Proceedings, 657-665. The final

publication is available at Springer via

https://doi.org/10.1007/978-3-319-70022-9_65

\title{
What Can We Learn from the Long-Term Users of a Social Robot?
}

\author{
Csaba Kertész ${ }^{1}$ and Markku Turunen ${ }^{1}$ \\ ${ }^{1}$ Tampere Unit for Computer-Human Interaction, University of \\ Tampere, Tampere, Finland \\ csaba.kertesz@ieee.org, markku.turunen@sis.uta.fi
}

\begin{abstract}
Despite the recent technological advances, long-term experiments with robots have challenges to keep the users interested after the initial excitement disappears. This paper explores the user expectations by analyzing the long-term owners of Sony AIBO who have been using these robots for years (heavy users). 78 participants filled an on-line questionnaire and their answers were inspected to discover the key needs of this user group. Quantitative and textual methods confirmed that the most-wanted skills were the interaction with humans and the autonomous operation. The integration with the AI agents and Internet services was important, but the long-term memory and learning capabilities were not that relevant for the participants as expected. The diverse preferences between robot skills led to the creation of a prioritized recommendation list to complement the design guidelines for social robots in the literature.
\end{abstract}

Keywords: questionnaire, user expectations, heavy users, Sony AIBO.

\section{Introduction}

Nowadays more and more social robots are introduced onto the market and the user expectations must be understood for the researchers to execute successful long-term experiments and for the companies to create sustainable business plans. Graaf et al [6] developed a theoretical foundation to describe the relationship between the owner and its robot over time. In the adoption and adaptation phases, the first experiences are gained with the robot at home. When the novelty effect fades away and the user expectations are met, daily routines are developed with the robot (incorporation phase). After six months, the owner gets emotionally attached to the robot as a personal object (identification phase) then the robot is finally accepted for long-term use and the owner becomes a heavy user. The ultimate goal in robotics is to reach this acceptance phase and keep the heavy users engaged with the robot for years. Although Nao and other humanoid robots are well-known from the news, their primary users are the research labs while Sony AIBO was the first successful social robot brand offered for private customers. This study focused on the latter robots because their owners are ordinary people forming the eventual target group of the social robotics to bring robots into the mainstream. The Sony robot dogs were so ahead of their time that a significant heavy user base is still reachable long after the discontinuation, therefore, a questionnaire was 
conducted with this user group to identify their long-term expectations. The literature examined the phases before the robot acceptance, but we do not have a broad understanding of the heavy users beyond the identification phase. This paper is a preliminary step to build this knowledge.

The human-robot interaction (HRI) field studied several robots in the past. A general observation was that the users have decreasing interest for the robots after their novelty effect fades away [5, 10]. To avoid losing attraction, social or other engaging capabilities must be identified to create robots for longer use. The long-term interaction was studied by Leite et al [8] in a survey of exploratory papers in health care, education, work and home settings. They admitted that the reviewed experiments were carried out with limited number of users and the purpose of the longer duration was to let the participants to get comfortable with the experimental conditions. Their results suggested that the people were happy to interact with the social robots for longer periods, but they proposed further analysis to confirm this hypothesis.

Graaf et al [7] researched with Nabaztag and Karotz robots to create guidelines for better user acceptance. They emphasized the importance of a clear purpose for the robot and the use context because the owners will abandon a robot without utility value. In this way, a truly social personality with interaction skills can differentiate a robot from other gadgets. The authors of [7] also warned the designers that they must consider the mere-exposure effect when the increased familiarity with the robotics technologies will reshape the robot acceptance inside the society over time. The same Karotz experiment was analyzed further in [14] and they found that the users did not reach the acceptance phase mainly caused by the end of novelty, unsatisfied needs, functional replacement (other device) and disappointment in the robot.

The HRI literature showed that the social robots must be designed carefully to engage the users. This study focuses on a domestic social robot, namely, the owners of Sony AIBO robots were analyzed. This product brand included quadruped autonomous entertainment robots which had a behavior-based architecture to exhibit a life-like impression. These robots can walk around the room, interact with the owner and switch between probabilistic state machines to show rich behaviors and engage the owners. Several papers studied AIBO in the past decade, but heavy users were never evaluated directly although Bartneck et al [1] studied the cross-cultural differences how people perceive AIBO after an interaction session and the on-line forums [4] were analyzed to investigate the relationship between the robots and their owners.

To the best knowledge of the authors, all previous researches in HRI lasted no more than one year and they usually organized short weekly or monthly sessions for the participant together with the robot [2,3]. Although some relevant results were gained from these past researches, but the subjects in this experiment lived with these social robots day by day for years. This paper explores the expectations of heavy users from a technical perspective what is different from the previous studies which investigated the user perception [6] and the reasons for abandoning robots before the identification phase [14]. Instead of asking the people how they perceive their robot or why they left them behind, the authors addressed in this study what kind of improvements do the participants expect to remain in acceptance phase? 


\section{Questionnaire}

A questionnaire was conducted to get the opinion of people about their Sony AIBO robots. Eight questions asked basic information of the participants (gender, age, home location, profession) and the robot ownership (length, usage frequency, model preference). The usage frequency question ensured that the participants had constant interactions with their robots thus they were part of the target group (heavy users). The following questions were related to the perception of their robots, how they feel about the existing software and which skills must be improved in AIBO. These questions had 9-point Likert-type items (anchors: 1 - Strongly disagree, 3 - Disagree, 5 - Neutral, 7 Agree, 9 - Strongly agree) and optional text fields were present where the participants could write additional information. A tendency was in the free-form answers that the participants left mostly technical feedback, therefore, two questions (wishes for skills and connectivity options) and the free-form answers were analyzed to characterize the long-term user expectations in this paper.

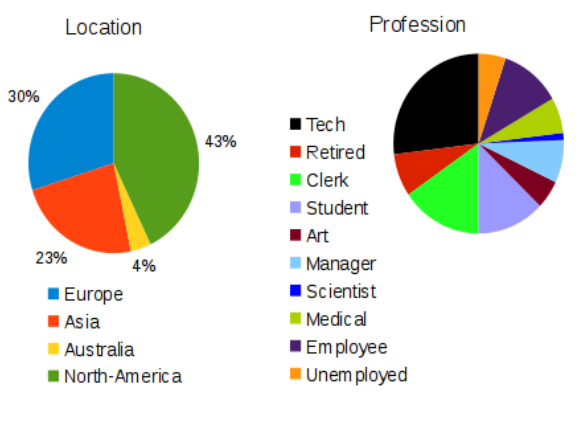

Fig. 1 The home location (1.a) and profession (1.b) of the AIBO customers who responded to the questionnaire.
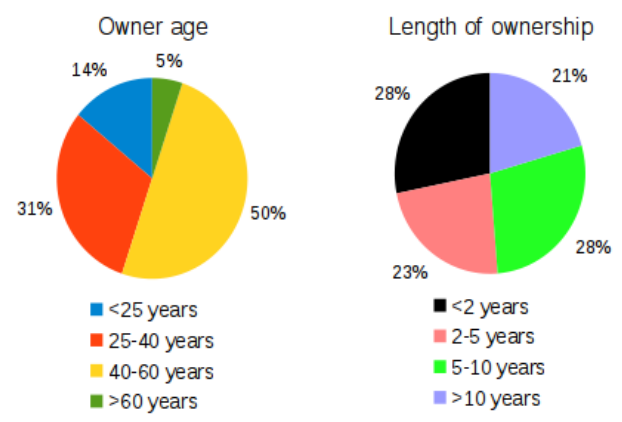

Fig. 2 The age (2.a) and length of ownership of the participants.

78 fillings were collected from the members of an English speaking on-line AIBO forum (http://aibo-life.org) what is similar to 70 in [6] and 17 Japanese participants were reached via Facebook ad campaign, similar to [11]. The questionnaire was completed by 57 male and 19 female, since two participants did not reveal their gender, with a ratio $73 \% / 24 \%$, similar to another on-line AIBO questionnaire with $64 \% / 36 \%$ in [1] and a robotics questionnaire had $61 \% / 39 \%$ in [13]. Although there was no question about the income and the wealth of the participants, the authors assume that this rate can be explained with the higher interest of the men in gadgets [12]. The technical enthusiasm was also reflected in the professions because most owners were e.g. engineers, software developers, technicians (27\% for Tech in Fig. 1.b) and other occupations were between 1-15\% in Fig. 1.b.

Since this study focused on robot consumers with more years of ownership, the participants bought and kept these robots after technology acceptance. 14\% of these owners were young adult (under 25 years) and their stories on the on-line forum given 
an insight of their intentions to buy these robots. Either they have got know AIBO in the recent years or they were children during the commercialization of AIBO and they could afford these robots after becoming an adult with income. Fig. 2.b shows high retention rate for $20 \%$ of the participants who kept their robots for more than 10 years, $51 \%$ had AIBO between 2-10 years, but $28 \%$ possessed AIBO for less than 2 years which is a high rate of newcomers.

\section{Result of Quantitative Analysis}

Two questions asked the owners about their technical expectations explicitly and both were composed of Likert-type items. Their consistency was verified with Cronbach's $\alpha$ coefficients $(0.91,0.81)$ thus there was sufficient trust in the overall reliability of the answers.

The average ratings for software improvements in descending order are shown in Fig. 3. Enhancing the dances (5.6), toy-like functions (3.4), the tricks with plastic toys of the robot (ball: 6.7, bone: 6.3) and dog-like behaviors (6.4) had low interest among the participants, most likely, because these features provide the entertainment aspect and the people are more eager to interact with their robots. The exceptionally low ratings of the toy-like function (3.4) can root in the expected anticipation that a robot must be intelligent and it is not a soulless toy. The human-robot interaction capabilities had the highest ratings: speech recognition (7.9), interacting (7.7), distinguish humans (7.7), emotion recognition (7.4), talking (7.1) and playing games (7.0). These skills shape a more valuable emotional connection for the owner towards the robot instead of watching repetitive entertainment behaviors. Although the autonomous features (navigation in rooms: 7.9, object recognition: 7.9) got high ratings, the participants wanted the robot moderately independent (6.8).

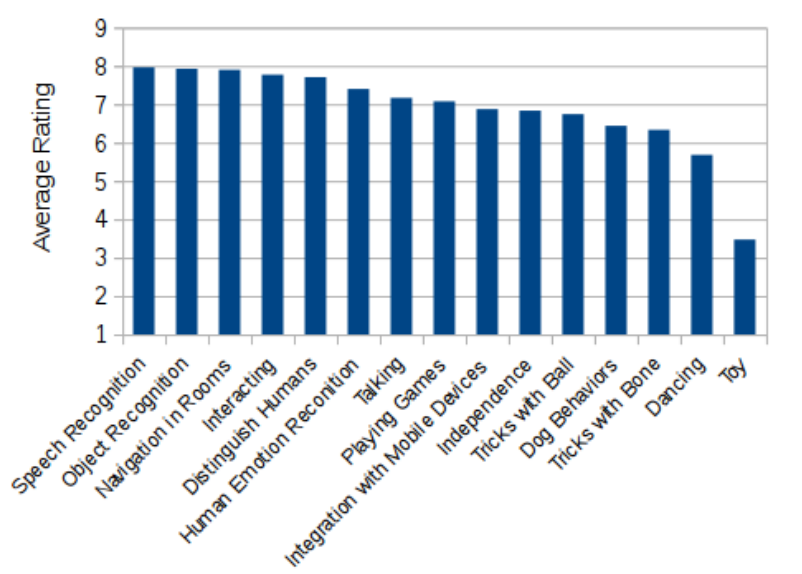

Fig. 3 Average ratings for improvements in the current robot software of Sony AIBO. 


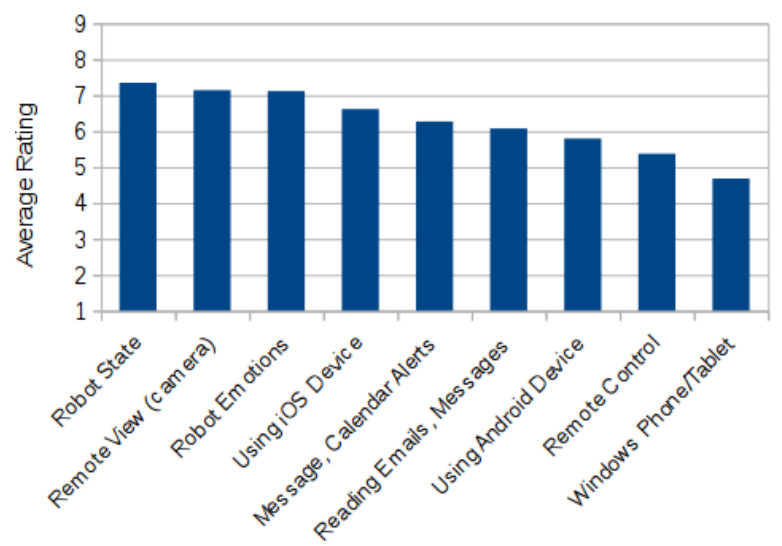

Fig. 4 Average ratings for wished connection options in a new software of Sony AIBO.

The results for connectivity options are shown in Fig. 4. The owners wished most to view robot state (7.3), emotion (7.1) and the camera (7.1), but controlling the robot remotely (5.3) was not interesting. Among the handheld devices, the participants would like to connect their bots to iOS devices (expensive products like robots) with the highest chance (6.6) while Android had a moderate result (5.7), and according to the low market share, Windows devices had the lowest score (4.6). Reading messages (6.2) and emails (6.0) had medium ratings.

\section{Result of Free-Form Answers}

The participants had the chance to give optional feedback in free form text without any directions. $60 \%$ of the Westerners provided this extra feedback with rich statements, nevertheless, the Japanese had a lower profile with $47 \%$ and their short answers were infrequent. Despite these data were free text, the participant given consistent answers by emphasizing certain robot skills or pointing out missing capabilities. To analyze the motives, the main points were extracted and counted in all answers like "votes". A list was created from the results in descending order by votes in Table 1 and the items with less than 5 votes were omitted.

The interaction is essential for social robots. Disappointed Pleo owners reported in [2] that "it would be more important to interact with you then wagging his tail in 28 different ways". Although AIBO has conversational and interaction skills to some extent, their enhancements were the most requested ( $1^{\text {st }}$ item in Table 1$)$, similar to the quantitative results in Fig. 3. Suggestions included extensions to the limited vocabulary (50-100 words) and the voice recognition performance was criticized to be far from perfect. With the wave of the AI agents in the smartphones (e.g. Siri, Cortana), people expect to include these technologies in the robots ("It would be great if Aibo could talk through a program like Siri or something."). The integration with Internet services $\left(5^{\text {th }}\right.$ item) is closely related to the conversational skill, it extends the local AI with live 
information from the web ("being able to ask him ... what the weather is today, etc. would be great.").

Table 1 Grouped results of the free form answers by the participants. The main points were extracted from the important statements of the owner feedback. Votes denote the occurrences of a main point in multiple answers.

\begin{tabular}{|c|l|c|}
\hline & Main Points & Votes \\
\hline 1 & Better conversational and interaction skills. & 20 \\
\hline 2 & Autonomous operation. & 18 \\
\hline 3 & Richer personality and new content (e.g motions) over time. & 16 \\
\hline 4 & Connectivity options for Apple/Windows/Android devices & 16 \\
\hline 5 & Integration with Internet services (e.g. email, weather, social, Siri). & 9 \\
\hline 6 & More learning capabilities, long-term memory. & 9 \\
\hline 7 & AIBO was ahead of its time. Lack of successor. & 6 \\
\hline 8 & Good face and object recognition. & 6 \\
\hline 9 & More settings to tweak the robot behaviors. & 6 \\
\hline 10 & Third-party software is better. & 5 \\
\hline 11 & Home automation integration. & 5 \\
\hline 12 & Remote control, house surveillance and protection. & 5 \\
\hline 13 & Self-charging. & \\
\hline
\end{tabular}

Another prioritized expectation for social robots is to operate autonomously $\left(2^{\text {nd }}\right.$ item), similar to Fig. 3. Albeit people love to interact with their robots, they also like to see their bots wandering around. Genibo, a later Korean robot dog model on the market, was criticized by their consumers that it constantly whines in one place, begs for the attention of its owner and it does not act too much on its own. This feedback exposes the importance of the autonomous activities of the social robots for long-term acceptance.

As the time passes by, people get bored with the same software and they expect new content to keep the amusement with their robots ( $3^{\text {rd }}$ item). Sony released their AIBO robots with a high price tag and there were no software updates over time. The $3^{\text {rd }}$ party developers got limited chances to build new applications for this brand, nonetheless, the participants praised these software over the official in the $10^{\text {th }}$ item. Nowadays, the success of the mobile app stores and the in-app purchase show that people are prepared to pay for new content if they are worth. The traditional business models can be 
extended with content purchase or monthly subscriptions to ensure the future commercial success for social robots.

The enhanced connectivity options ( $4^{\text {th }}$ item) was in tie with the previous item. The participants were reasonable to desire wireless links to their new gadgets after the technological evolution in the past decade. This result is aligned with Fig. 3 where the connectivity skill was ranked after the interaction and autonomous skills.

One surprising finding was that the owners ranked the learning capabilities, memory function, face and object recognition $\left(5^{\text {th }}\right.$ and $8^{\text {th }}$ items $)$ half less important than the top items. Although these abilities are necessary for humans to perceive real intelligence, the utility of a social robot for its owner is focused on building an emotional attachment with the interaction skills.

Some tech-savvy features were ranked to the lowest. The advanced settings for the robot $\left(9^{\text {th }}\right.$ item $)$, the home automation integration $\left(11^{\text {th }}\right.$ item $)$ and the house surveillance $\left(12^{\text {th }}\right.$ item) were present in Table 1 , but they had low priority. This outcome suggests for the designers to invent the appearance and tech features according to the specific purpose to maximize the utility.

Some conflicting requests were interesting which are not listed in Table 1 . The AIBO software mix the dog-like behaviors with sound effects and the verbal conversations with humans. On one hand, these robots resemble an animal by their appearance and some people wanted to disable the more intelligent features ("I would ... turn my ERS7 into a pet dog, no dancing, or talking"). On the other hand, some users would like the opposite, dropping the dog behaviors and including more anthropomorphic features to see a conversational autonomous agent ("Maybe make a new software ... with ... no dog like actions. And just purely interacts with human speaking.").

The Sony robots had a sophisticated software in the $2000 \mathrm{~s}$, but it was far what average people would call artificial intelligence. This chapter given an insight how the heavy users positioned the important skills for their social robot and how the technological evolution influenced these preferences.

\section{Discussion}

\subsection{Social Robot Design}

Two papers proposed guidelines to improve the design of social robots for long-term human-robot interactions. Leite et al [8] presented a good review of this problem with a detailed discussion by accumulating the experiences of different robots in the research literature while [7] expressed their recommendations on a higher level. It was proposed in [7] to create a clear purpose for the robot because this important factor can lead to acceptance by their owners, but we argue that the clear purpose is not enough. If the robot cannot surpass the competing devices in our life in utility value, people will leave and turn to other machines [6]. Based upon the quantitative analysis (Chapter 3) and the ranked textual feedback (Chapter 4), the authors propose the following recommendations in descending priority to complement the past works [7, 8]:

- Design the robot appearance according to its capabilities to avoid the uncanny valley [9]. Sony AIBO was successful because it resembled an 
animal, walked around and had interactions with humans according to the ads. The owners expected less intelligence from these robot dogs than a humanoid robot [5]. Pleo owners were disappointed in [2] when they realized that their robots have legs, but they cannot walk without a trigger.

- The interaction and conversational skills were found the most important by the users. This is in accordance with the third recommendation in [7], the perceived sociability is a key factor for the users to accept the robot.

- Integrating the latest web services and conversational agents into the robot enhances the user experience. When intelligent applications (e.g. Siri, Alexa) are available in handsets or computers, people expect to have similar built-in skills in their robots.

- The owners expect autonomy, self-charging from a mobile robot as well as a remote application to interact with the robot and check its status.

- The people gets bored quickly with the repetitive behaviors [10], but if the robot is attractive enough, the owners keep the robots for a long time. The participants of this experiment verify this assumption. However, the users expect new behaviors constantly (Chapter 4) and this opportunity can be turned into a business strategy for social robots to charge regular fee per content update, similar to the mobile applications. This approach with a broad userbase can create a sustainable revenue for a robotics company.

- The learning and memory skills are hard problems in the artificial intelligence. Leite et al [8] stated that the benefits of memory is unclear in the long-term interaction, nevertheless, AIBO users explicitly asked this skill. Although this feature is important, it can be prioritized less than the communication and interactions skills according to Chapter 4.

- The target group of the social robots is broad from the teenagers to the pensioners (Fig. 2) although 50\% were between 40-60 years.

\subsection{Limitations}

Despite of the participants were recruited on a special internet forum and Facebook, only 78 active Sony AIBO owners were reached, but the authors believe that sample size was reasonable compared to 230 in [1] and 41 in [13] considering that conducting our survey was long after the product discontinuation. The sampling was not representative for the general public, but the participants could provide a good indication about the typical users of entertainment robots and even beyond this group since Bartneck et al found in [1] that owning a Sony AIBO did not result significantly different scores on their NARS questionnaire.

Since these robots were commercial, this study was essential to analyze the heavy users of an expensive robot from the market. The robots in past experiments were given to participants on a voluntary basis for free $[2,7]$.

\subsection{Conclusion}

The Westerner and Japanese heavy users of Sony robot dogs were studied with a questionnaire in this paper after 10 years of the product discontinuation. Since these 
people owned their robots for years after the initial "wow" moment faded out, they were already in the robot acceptance phase. Despite Sony AIBO was ahead of its time, it exhibited several mechanical and software limitations, and definitely, the heavy users were not satisfied with the robot capabilities after many years without software updates. Both the quantitative analysis and the free-form text answers suggested that the mostwanted improvement was the interaction skills with humans, followed by the autonomous operations. The participants was not interested too much in the entertainment aspects, remote control or self-charging, but they would like to connect their robot with handheld devices and modern Internet services. It was surprising that the learning capabilities and long-term memory were moderately important for the users. After the questionnaire analysis, recommendations were written for social robot design to complement the initial guidelines in the literature [7, 8].

The future work can include similar analysis with heavy users of other robots and it can be worth to compare our results with different robot appearances or personalities.

Acknowledgements. The authors want to say thanks to all enthusiastic members of the aibo-life.org forums to fill out our questionnaire.

\section{References}

1. Bartneck, C., Suzuki, T., Kanda, T., Nomura, T.: The influence of people's culture and prior experiences with AIBO on their attitudes towards robots. Journal of AI \& Society, 21(1-2), 217-230 (2007)

2. Fernaeus, Y., Håkansson, M., Jacobsson, M., Ljungblad, S.: How do you play with a robotic toy animal?: A long-term study of pleo. In: Proc. of 9th Intl. Conf. on Interaction Design and Children, pp 39-48 (2010)

3. François, D., Powell, S., Dautenhahn, K.: A long-term study of children with autism playing with a robotic pet: taking inspirations from non-directive play therapy to encourage children's proactivity and initiative-taking. Journal of Interactive Studies 10(3), 324-373 (2009)

4. Friedman, B., Kahn, P. H., Hagman, J.: "Hardware companions?': What online AIBO discussion forums reveal about the human-robotic relationship. CHI Letters 5(1), 273-280 (2003)

5. Gockley, R., Bruce, A., Forlizzi, J., Michalowski, M., Mundell, A., Rosenthal, S., Sellner, B., Simmons, R., Snipes, K., Schultz, A., Wang, J.: Designing robots for long-term social interaction. In: Proc. of IEEE/RSJ Intl. Conf. on intelligent robots and systems (IROS), pp 1338-1343 (2005)

6. Graaf., M. M., Ben Allouch, S., Dijk, J. A.: Long-term evaluation of a social robot in real homes. In: Proc. of 3rd Intl. Symposium on New Frontiers in Human-Robot Interaction (AISB), (2014)

7. Graaf, M. M., Ben Allouch, S., Dijk, J.A.: Long-term acceptance of social robots in domestic environments. AAAI, (2016)

8. Leite, I., Martinho, C., Paiva, A.: Social robots for long-term interaction: a survey. Internation Journal of Social Robotics 5(2), 291-308 (2013)

9. Mori, M.: The uncanny valley. Energy 7(4):33-35 (1970)

10. Salter, T., Dautenhahn, K., Bockhorst, R.: Robots moving out of the laboratory-detecting interaction levels and human contact in noisy school environments. In: Proc. of 13th IEEE 
Intl. workshop on robot and human interactive communication (ROMAN), pp 563-568 (2004)

11. Samuels, D., Zucco, C.: Using Facebook as a subject recruitment tool for surveyexperimental research. Social Science Research Network (2012)

12. Yoldas, S.: A Research about Buying Behaviours of Online Customers. MSc Thesis, University of Roehampton (2012)

13. Haring, K. S., Mougenot, C., Fuminori, O.N.O., Watanabe, K.: Cultural differences in perception and attitude towards robots. International Journal of Affective Engineering 13(3), 149-157 (2014)

14. Graaf, M.M., Allouch, S.B., Dijk, J.A.: Why Do They Refuse to Use My Robot?: Reasons for Non-Use Derived from a Long-Term Home Study. In: Proc. of Intl. Conf. on HumanRobot Interaction, pp 224-233 (2017) 